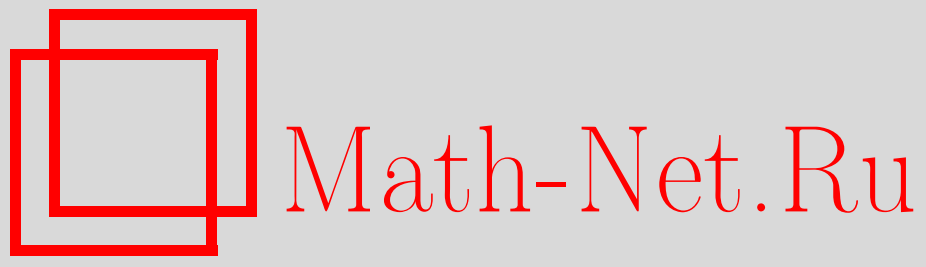

Р. С. Исмагилов, Индуктивные пределы групп диффеоморфизмов, сохраняющих площадь, Функи. анализ и его прил., 2003, том 37, выпуск 3, 36-50

DOI: https://doi.org/10.4213/faa156

Использование Общероссийского математического портала MathNet.Ru подразумевает, что вы прочитали и согласны с пользовательским соглашением

http://www . mathnet.ru/rus/agreement

Параметры загрузки:

IP: 54.172 .240 .79

26 апреля 2023 г., 13:25:36

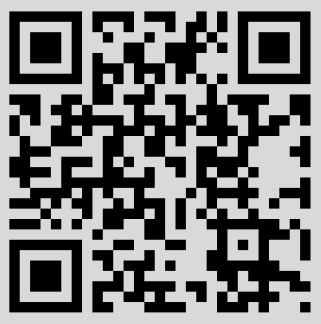


Функциональный анализ и его приложения

2003, т. 37, вып. 3, с. 36-50

УДК 517.54

\title{
Индуктивные пределы групп диффеоморфизмов, сохраняющих площадь*
}

\author{
(c) 2003. Р. С. ИСМАГИЛОВ
}

\section{Введение}

Напомним определение индуктивного предела в теории групп [1]. Пусть $\left\{G_{\alpha}\right\}$ - набор групп и для любых $\alpha, \beta$ дан набор (возможно, пустой) гомоморфизмов $i_{\alpha, \beta}: G_{\alpha} \rightarrow G_{\beta}$. Индуктивный предел семейства $\left\{G_{\alpha}, i_{\alpha \beta}\right\}-$ это группа $\Gamma$ и набор гомоморфизмов $s_{\alpha}: G_{\alpha} \rightarrow \Gamma$, удовлетворяющие следующим условиям: 1) $s_{\alpha}=s_{\beta}$ 。 $i_{\alpha \beta}$ для всех $\left.\alpha, \beta, i_{\alpha \beta} ; 2\right)$ для любой группы $\Gamma^{\prime}$ и гомоморфизмов $f_{\alpha}: G_{\alpha} \rightarrow \Gamma^{\prime}$, удовлетворяющих аналогичным условиям, существует единственный гомоморфизм $F: \Gamma \rightarrow \Gamma^{\prime}$, такой, что $f_{\alpha}=F \circ s_{\alpha}$ для всех $\alpha$.

Аналогичным образом определяется индуктивный предел семейства алгебр (в частности, алгебр Ли).

Опишем предмет этой статьи. Рассмотрим компактную ориентируемую поверхность (без края) с заданной формой площади. Для каждой координатной окрестности $\sim \mathbb{R}^{2}$ (здесь и далее $\sim$ означает диффеоморфность) возьмем группу сосредоточенных в ней диффеоморфизмов, сохраняющих площадь. Для двух окрестностей, одна из которых содержится в другой вместе с замыканием, имеем очевидный гомоморфизм - включение одной группы в другую. Получаем семейство групп и гомоморфизмов между ними. Еще одно семейство получается, если в каждой из этих групп взять нормальную подгруппу коразмерности 1 (ее коммутант). Мы опишем индуктивные пределы этих двух семейств групп. Больший интерес представляет второе из этих семейств (ему посвящена основная часть статьи). Для обоих семейств решение задачи об индуктивном пределе приводит к центральному расширению группы диффеоморфизмов, которая порождена диффеоморфизмами, сосредоточенными в координатных окрестностях и сохраняющими площадь. При этом для первого семейства это расширение расщепляется, а для второго - нет.

В §8 приведены некоторые следствия; их можно отчасти рассматривать как мотивировки предпринятого исследования. Прежде всего, мы извлекаем из указанных результатов некоторые сведения о строении уже упомянутой группы диффеоморфизмов. Диффеоморфизмы, сосредоточенные в координатных окрестностях, - это образующие рассматриваемой группы. Обратимся к соотношениям между ними. Имеем, прежде всего, всевозможные соотношения между диффеоморфизмами, сосредоченными в одной координатной окрестности; эти соотношения можно назвать «локальными». Оказывается, однако, что их недостаточно для задания нашей группы; другими словами, абстрактная группа, заданная указанными образующими и соотношениями, изоморфна не упомянутой группе

*Работа поддержана РФФИ, грант 01-01-00490 
диффеоморфизмов, а ее центральному расширению. Чтобы «убить» центр (и, таким образом, получить саму группу диффеоморфизмов), необходимо добавить к очевидным «локальным» соотношениям некоторую совокупность (желательно, достаточно простую) «глобальных» соотношений. Это сделано в §8. В этом параграфе отмечены также точные последовательности, связывающие рассматриваемые группы диффеоморфизмов.

\section{§1. Группы диффеоморфизмов, сохраняющих форму площади}

В этом параграфе собраны известные сведения, обычно излагаемые в гораздо большей общности [2-6]. Мы отберем только то, что необходимо для дальнейшего.

1.1. Поверхность и группа $\boldsymbol{D}\left(\boldsymbol{X}, \boldsymbol{\omega}^{\mathbf{2}}\right)$. Пусть $X-$ поверхность, т. е. двумерное (возможно, несвязное) многообразие класса $C^{\infty}$ (без края). Перечислим необходимые термины и обозначения.

Кругом в $X$ мы называем образ круга в евклидовой плоскости при вложении ее в $X$. Окружность, интервал и отрезок определяются аналогично.

Пусть $E^{k}(X), k=1,2$, - пространство внешних $k$-форм с компактным носителем, $d-$ внешнее дифференцирование, $Z^{k}(X)=\operatorname{ker} d \mid E^{k}(X), B^{k}(X)=$ $d\left(E^{k-1}(X)\right), \quad k=1,2, H_{c}^{1}(X, \mathbb{R})=Z^{1}(X) / B^{1}(X)$ (пространство когомологий с компактным носителем; для компактного многообразия $X$ пишем $\left.H^{1}(X, \mathbb{R})\right)$. Класс когомологий формы $a \in Z^{1}(X)$ обозначаем через $[a]$, а действие диффеоморфизма $g$ на форму $\gamma-$ через $g \gamma$.

Существуют такие непрерывные линейные отображения $J^{k}=J_{X}^{k}: B^{k}(X) \rightarrow$ $E^{k-1}(X), k=1,2$, что $d \circ J^{k}(\xi)=\xi$ при $\xi \in B^{k}(X)$. Если даны круги $U_{0}, U$ и $\bar{U}_{0} \subset U$, то отображение $J^{2}$ можно выбрать так, что $J^{2}\left(B^{2}\left(U_{0}\right)\right) \subset E^{1}(U)$ (см. приложение, п. b)). Для любой области $Y \subset X, Y \sim \mathbb{R}^{2}$, возьмем линейное непрерывное отображение $J_{Y}^{2}: B^{2}(Y) \rightarrow E^{1}(Y)$, такое, что $d \circ J_{Y}^{2}(\xi)=\xi$ при $\xi \in B^{2}(Y)$.

Переходим к группам. Пусть $X$ ориентируемо и задана всюду невырожденная 2 -форма $\omega^{2}$ (форма площади). Пусть $D\left(X, \omega^{2}\right)-$ группа диффеоморфизмов $x \mapsto x g$, для которых существует такое семейство диффеоморфизмов $\left\{g_{t}\right\}$, $0 \leqslant t \leqslant 1$, непрерывно зависящее от $t$, что $g_{t} \omega^{2}=\omega^{2}$ для всех $t, g_{1}=g, g_{0}=e$ и (в случае некомпактного $X$ ) все $g_{t}$ тождественны вне фиксированного компакта (e - тождественный диффеоморфизм). Обозначим через $D_{*}\left(X, \omega^{2}\right)$ подгруппу, порожденную всеми подгруппами $D\left(Y, \omega^{2}\right)$, где $Y \subset X, Y \sim \mathbb{R}^{2}$.

Для открытого подмногообразия $Y \subset X$ группа $D\left(Y, \omega^{2}\right)$ естественно вкладывается в $D\left(X, \omega^{2}\right)$.

1.2. Некоторые отображения. а) Пусть $X$ связно и некомпактно. Тогда $\omega^{2}=$ $d \omega^{1}$ для некоторой формы $\omega^{1}$. Если $g \in D\left(X, \omega^{2}\right)$, то форма $g \omega^{1}-\omega^{1}$ замкнута и имеет компактный носитель. Это приводит к гомоморфизму $\alpha: D\left(X, \omega^{2}\right) \rightarrow$ $H_{c}^{1}(X, \mathbb{R})$. Справедливо равенство $\operatorname{ker} \alpha=D_{*}\left(X, \omega^{2}\right)$.

Опишем отображение $\alpha$ иначе. С этой целью возьмем элемент $g \in D\left(X, \omega^{2}\right)$, непрерывное (по $t)$ семейство $g_{t} \in D\left(X, \omega^{2}\right), t \in[0,1], g_{0}=e, g_{1}=g$, и локально конечный 1 -цикл $l$ в $X$. Определим поток диффеоморфизма $g$ через цикл $l$ равенством $\theta(l, g)=\int_{\left\{l g_{t}\right\}} \omega^{2}$, где $\left\{l g_{t}\right\}$ - это 2 -цепь, «заметаемая» 1 -циклами $l g_{t}, 0 \leqslant t \leqslant 1$ (более формальное определение излишне). Указанный интеграл не 
зависит от выбора семейства $\left\{g_{t}\right\}$ и обращается в нуль, если $l-$ граница 2 -цепи. Возникает отображение $D\left(X, \omega^{2}\right) \rightarrow H_{c}^{1}(X, \mathbb{R})$. Это и есть $\alpha$.

b) Пусть снова $X$ некомпактно и связно. Тогда определен гомоморфизм $\beta(X, \cdot): D_{*}\left(X, \omega^{2}\right) \rightarrow \mathbb{R}$ по формуле $\beta(X, g)=\int_{X} \omega^{1} \wedge g \omega^{1}$. Положим $D^{0}\left(X, \omega^{2}\right)=$ $\operatorname{ker} \beta(X, \cdot)$.

с) Пусть $X$ есть конечное объединение связных некомпактных компонент $X_{i}$. Определим подгруппу $D^{0}\left(X, \omega^{2}\right) \subset D\left(X, \omega^{2}\right)$ как $\prod_{i} D^{0}\left(X_{i}, \omega^{2}\right)$. Итак, $D^{0}\left(X, \omega^{2}\right)$ $\subset D_{*}\left(X, \omega^{2}\right) \subset D\left(X, \omega^{2}\right)$.

d) Пусть $X$ связно и компактно. Группа $D\left(X, \omega^{2}\right)$ локально стягиваема. Это позволяет определить для диффеоморфизмов из $D\left(X, \omega^{2}\right)$, близких к единице, поток через 1 -цикл (как и для некомпактного $X$ ). Возникает локальный гомоморфизм $\alpha: D\left(X, \omega^{2}\right) \rightarrow H^{1}(X, \mathbb{R})$.

Вот еще одна реализация отображения $\alpha$. Возьмем точку $x_{0} \in X$ и такую 1 -рорму $\omega^{1}$ на $X \backslash\left\{x_{0}\right\}$, что $\omega^{2}=d \omega^{1}$ на $X \backslash\left\{x_{0}\right\}$. Возьмем круг $U_{0} \ni x_{0}$ и такую окрестность единицы $W=W^{-1} \subset D\left(X, \omega^{2}\right)$, что $x_{0} g \in U_{0}$ при $g \in W$. Для любого $g \in W$ имеем замкнутую 1 -форму $g \omega^{1}-\omega^{1}$ на $X \backslash\left\{x_{0}, x_{0} g^{-1}\right\}$. Существует (на всем $X$ ) замкнутая 1 -форма $\phi_{g}$, совпадающая с $g \omega^{1}-\omega^{1}$ вне $\bar{U}_{0}$ и равная нулю в окрестностях точек $x_{0}, x_{0} g^{-1}$. Класс $\left[\phi_{g}\right]$ не зависит от произвола в выборе формы $\phi_{g}$. Отображение $W \rightarrow H^{1}(X, \mathbb{R}), g \mapsto\left[\phi_{g}\right]$, совпадает с $\alpha$ в некоторой окрестности единицы.

Для дальнейшего (см. разд. 2.2) подчиним форму $\phi_{g}$ следующему ограничению:

$$
\int_{X} \phi_{g} \wedge \omega^{1}=0
$$

(форма, стоящая под знаком интеграла, определена, ибо форма $\phi_{g}$ равна нулю в окрестности точки $\left.x_{0}\right)$.

Отныне поверхность $X$ связна, компактна, ориентируема и

$$
\text { род поверхности } X \text { больше двух. }
$$

(Таким образом, $X-$ не тор и не сфера.)

Если выполняется условие $(*)$, то группа $D\left(X, \omega^{2}\right)$ стягиваема (приложение, п. а)). Поэтому локальный гомоморфизм $\alpha$ продолжается до гомоморфизма $D\left(X, \omega^{2}\right) \rightarrow H^{1}(X, \mathbb{R})$. При этом $D_{*}\left(X, \omega^{2}\right)=\operatorname{ker} \alpha$.

Завершим этот раздел следующим уточнением терминологии. Локальныци гомоморфизм (он уже появлялся выше) топологической группы $G$ в группу $A$ задается окрестностями единицы $W_{0}=W_{0}^{-1} \subset W=W^{-1} \subset G$ и отображением $f: W \rightarrow A$, такими, что $W_{0} \cdot W_{0} \subset W$ и $f\left(g_{1} g_{2}\right)=f\left(g_{1}\right) f\left(g_{2}\right)$ при $g_{i} \in W_{0}$, $i=1,2$. Аналогично определяются локальные коциклы на $G$ со значениями в $A$.

\section{§2. Центральное расширение группы $D_{*}\left(X, \omega^{2}\right)$ и описание результата}

2.1. Постановка задачи. Пусть $X$ - компактная поверхность (без края) с формой площади $\omega^{2}$. Рассмотрим семейство групп $\left\{D^{0}\left(Y, \omega^{2}\right), Y \subset X, Y \sim \mathbb{R}^{2}\right\}$, с очевидными включениями $D^{0}\left(Y_{1}, \omega^{2}\right) \hookrightarrow D^{0}\left(Y_{2}, \omega^{2}\right)$ при $\bar{Y}_{1} \subset Y_{2}$. Требуется найти индуктивный предел семейства $\left\{D^{0}\left(Y, \omega^{2}\right)\right\}$. Ответ описан в п. 2.2; доказательства приводятся в $\$ 3-6$. Аналогичная (более простая) задача решается в $§ 7$ и для семейства $\left\{D\left(Y, \omega^{2}\right), Y \sim \mathbb{R}^{2}\right\}$. 
Некоторые вопросы этого круга рассмотрены в работах автора $[12,13]$ (в [13] рассматриваются алгебры Ли векторных полей на многообразии).

2.2. Центральное расширение группы $D_{*}\left(X, \omega^{2}\right)$; описание результатов. Всюду в этом разделе $X$ означает компактную поверхность (без края) с формой площади $\omega^{2}$.

Пусть $V\left(X, \omega^{2}\right)$ - алгебра Ли векторных полей, сохраняющих форму $\omega^{2}$, a $V_{*}\left(X, \omega^{2}\right)$ - подалгебра полей, представимых в виде конечной суммы слагаемых из $V\left(X, \omega^{2}\right)$, сосредоточенных в областях $\sim \mathbb{R}^{2}$. Алгебра Ли $V_{*}\left(X, \omega^{2}\right)$ имеет центральное расширение посредством $H^{1}(X, \mathbb{R})$ (см. [6], а также [7] и содержащиеся там ссылки на литературу). Это расширение задается 2-коциклом на $V_{*}\left(X, \omega^{2}\right)$, который определяется следующим образом. Возьмем непрерывный линейный проектор $P: E^{1}(X) \rightarrow Z^{1}(X)$ (его нетрудно построить). Возьмем любые $\xi_{i} \in V_{*}\left(X, \omega^{2}\right)$ с гамильтонианами $f_{\xi_{i}}, i=1,2$, и поставим им в соответствие элемент $\left[P\left(f_{\xi_{1}} d f_{\xi_{2}}\right)\right]$. Это и есть нужный 2 -коцикл. В работе [13] показано, что это расширение есть индуктивный предел семейства алгебр Ли векторных полей, сосредоточенных в координатных окрестностях и имеющих сосредоточенные в них гамильтонианы с нулевым интегралом. Описанный 2-коцикл можно «поднять» с алгебры Ли на группу $D_{*}\left(X, \omega^{2}\right)$ (благодаря стягиваемости этой группы). Общая конструкция «поднятия» дает весьма громоздкий 2-коцикл; можно, однако (после ряда попыток) заменить его когомологичным и более простым. Сейчас мы его опишем.

Возьмем точку $x_{0} \in X$ и круги $U_{0}, U$, такие, что $x_{0} \in U_{0}, \bar{U}_{0} \subset U$. Возьмем в $D_{*}\left(X, \omega^{2}\right)$ такие окрестности единицы $W_{0}, W$, что $x_{0} g \in U_{0}$ при $g \in W$ и $W_{0} W_{0} W_{0} \subset W$. Ниже используются отображения $J^{k}, J_{Y}^{2}, k=1,2, Y \sim \mathbb{R}^{2}$, указанные в разд. 1.1; отображение $J^{2}$ выберем таким, что $J^{2}\left(B^{2}\left(U_{0}\right) \subset E^{1}(U)\right)$. Сейчас мы построим локальный 2 -коцикл со значениями в $H^{1}(X, \mathbb{R})$, определенный на множестве $W \times W$.

Форма $\phi_{g}$, введенная в п. d) разд. 1.2 , точна при $g \in W$; в силу равенства (1) точна и форма $\omega^{1} \wedge \phi_{g}$. Это позволяет определить 1 -форму

$$
\rho\left(g_{1}, g_{2}\right)=J^{1}\left(\phi_{g_{1}}\right) \cdot g_{1} \phi_{g_{2}}+J^{2}\left(\left(g_{1} \omega^{1}-\phi_{g_{1}}\right) \wedge g_{1} \phi_{g_{2}}\right)-g_{1} J^{2}\left(\omega^{1} \wedge \phi_{g_{2}}\right),
$$

$g_{1} \in W, g_{2} \in W$.

Без труда доказывается, что она замкнута; обозначим соответствующий класс когомологий через $R\left(g_{1}, g_{2}\right)$.

Еще одно приготовление к формулировке результатов. Если $Y \subset X, Y \sim \mathbb{R}^{2}$, $g \in D^{0}\left(Y, \omega^{2}\right)$, то 1 -форма $\left(J^{2}-J_{Y}^{2}\right)\left(\omega^{1} \wedge \phi_{g}\right)$ очевидным образом замкнута; обозначим через $C_{Y}(g)$ соответствующий класс когомологий.

Теорема 1. (а) Построенное отображение $\left(g_{1}, g_{2}\right) \mapsto R\left(g_{1}, g_{2}\right)$ есть локальный 2 -коцикл на $D_{*}\left(X, \omega^{2}\right)$ со значениями в $H^{1}(X, \mathbb{R})$, m. е.

$$
R\left(g_{1}, g_{2}\right)+R\left(g_{1} g_{2}, g_{3}\right)=R\left(g_{1}, g_{2} g_{3}\right)+R\left(g_{2}, g_{3}\right) \quad \text { nрu } g_{1}, g_{2} \in W_{0} .
$$

Оно продолжается до 2-коцикла на всей группе $D_{*}\left(X, \omega^{2}\right)$ (сохраним для продолжения обозначение $\left.R\left(g_{1}, g_{2}\right)\right)$.

(b) Eсли $Y \subset X, Y \sim \mathbb{R}^{2}$, mо $R\left(g_{1}, g_{2}\right)=C_{Y}\left(g_{1} g_{2}\right)-C_{Y}\left(g_{1}\right)-C_{Y}\left(g_{2}\right), g_{i} \in$ $W_{0} \cap D^{0}\left(Y, \omega^{2}\right), i=1,2$.

Утверждение (a) этой теоремы дает центральное расширение

$$
0 \rightarrow H^{1}(X, \mathbb{R}) \rightarrow E\left(X, \omega^{2}\right) \stackrel{\pi}{\longrightarrow} D_{*}\left(X, \omega^{2}\right) \rightarrow 0,
$$


где группа $E\left(X, \omega^{2}\right)$ - это множество $D_{*}\left(X, \omega^{2}\right) \times H^{1}(X, \mathbb{R})$ с групповой операцией $\left(g_{1}, a_{1}\right)\left(g_{2}, a_{2}\right)=\left(g_{1} g_{2}, a_{1}+a_{2}+R\left(g_{1}, g_{2}\right)\right)$.

Согласно утверждению $(\mathrm{b})$, коцикл $\left(g_{1}, g_{2}\right) \mapsto R\left(g_{1}, g_{2}\right)$ является кограницей в некоторой окрестности единицы группы $D^{0}\left(Y, \omega^{2}\right)$. Так как эта группа стягиваема, то утверждение (b) выполняется на ней всюду для некоторого (однозначно определенного) продолжения отображения $C_{Y}$; мы сохраним для этого продолжения обозначение $C_{Y}$. Итак, над подгруппой $D^{0}\left(Y, \omega^{2}\right)$ расширение (4) расщепляется посредством гомоморфизма

$$
s_{Y}: D^{0}\left(Y, \omega^{2}\right) \rightarrow E\left(X, \omega^{2}\right), \quad g \mapsto\left(g, C_{Y}(g)\right) .
$$

Как уже сказано, расширение (4) получается из известного расширения алгебры Ли стандартным «поднятием» на группу, а потому, строго говоря, не ново. Наш основной результат - это выяснение следующего свойства расширения (4).

Теорема 2. Группа $E\left(X, \omega^{2}\right)$ с гомоморфизмами (5) есть индуктивный предел семейства $\left\{D^{0}\left(Y, \omega^{2}\right), Y \subset X, Y \sim \mathbb{R}^{2}\right\}$.

Наконец, рассмотрим семейство $\left\{D\left(Y, \omega^{2}\right), Y \sim \mathbb{R}^{2}\right\}$.

Tеорема 3. Групna $D_{*}\left(X, \omega^{2}\right) \times \mathbb{R}$, в которую группы $D\left(Y, \omega^{2}\right), Y \subset X$, $Y \sim \mathbb{R}^{2}$, вложень по правилу $g \mapsto(g, \beta(Y, g))$, есть индуктивный предел семейства $\left\{D\left(Y, \omega^{2}\right), Y \sim \mathbb{R}^{2}\right\}$.

Напомним, что мы исключили из рассмотрения сферу и тор. Отметим лишь, что для $X=S^{2}$ задача об индуктивном пределе (для обоих семейств $\left\{D^{0}\left(Y, \omega^{2}\right)\right\}$, $\left.\left\{D\left(Y, \omega^{2}\right)\right\}\right)$ решается универсальной накрывающей группы $D\left(S^{2}, \omega^{2}\right)$.

\section{§3. Доказательство теоремы 1}

1) Докажем равенство (3). Для этого достаточно проверить, что форма

$$
\rho\left(g_{1}, g_{2}\right)+\rho\left(g_{1} g_{2}, g_{3}\right)-\rho\left(g_{1}, g_{2} g_{3}\right)-g_{1} \rho\left(g_{2}, g_{3}\right), \quad g_{i} \in W_{0}, i=1,2,3,
$$

точна. Простое вычисление приводит эту форму к виду

$$
\begin{aligned}
& J^{1}\left(\phi_{g_{1}}\right) \cdot g_{1}\left(\phi_{g_{2}}-\phi_{g_{2} g_{3}}\right)+\left(J^{1}\left(\phi_{g_{1} g_{2}}\right)-g_{1} J^{1}\left(\phi_{g_{2}}\right)\right) \cdot g_{1} g_{2} \phi_{g_{3}} \\
& \quad+J^{2}\left(g_{1}\left(\phi_{g_{2}}-\phi_{g_{2} g_{3}}\right) \wedge\left(\phi_{g_{1}}-g_{1} \omega^{1}\right)+g_{1} g_{2} \phi_{g_{3}} \wedge\left(\phi_{g_{1} g_{2}}-g_{1} g_{2} \omega^{1}\right)\right) \\
& \quad+g_{1} J^{2}\left(\omega^{1} \wedge\left(\phi_{g_{2} g_{3}}-\phi_{g_{2}}\right)+\left(\phi_{g_{2}}-g_{2} \omega^{1}\right) \wedge g_{2} \phi_{g_{3}}\right) .
\end{aligned}
$$

Преобразуем эту форму, отбрасывая слагаемые, которые либо точны, либо сосредоточены в области $U$. Легко видеть, что формы, находящиеся под знаком отображения $J^{2}$, сосредоточены в области $U_{0}$. В силу свойств отображения $J^{2}$ слагаемые в $(7)$, содержащие $J^{2}$, сосредоточены в $U$. Отбросим их. Далее, заменив (в первом слагаемом из (7)) $\phi_{g_{2}}-\phi_{g_{2} g_{3}}$ на $-g_{2} \phi_{g_{3}}$, мы изменяем (7) лишь в области $U$. Функция $g_{1} J^{1}\left(\phi_{g_{2}}\right)$ (из второго слагаемого в $(7)$ ) отличается от функции $J^{1}\left(g_{1} \phi_{g_{2}}\right)$ на постоянную, ибо дифференциалы этих функций равны. Поэтому, заменив $g_{1} J^{1}\left(\phi_{g_{2}}\right)$ на $J^{1}\left(g_{1} \phi_{g_{2}}\right)$, мы изменяем форму (7) на точное слагаемое вида $\lambda g_{1} g_{2} \phi_{g_{3}}, \lambda \in \mathbb{R}$. Эти замены приводят к форме $\tau^{1}=J^{1}\left(-\phi_{g_{1}}+\phi_{g_{1} g_{2}}-g_{1} \phi_{g_{2}}\right) \cdot g_{1} g_{2} \phi_{g_{3}}$. Форма, стоящая под знаком $J^{1}$, сосредоточена в $U_{0}$; поэтому функция $J^{1}\left(-\phi_{g_{1}}+\phi_{g_{1} g_{2}}-g_{1} \phi_{g_{2}}\right)$ постоянна вне области $U$. Таким образом, $\tau^{1}$ пропорциональна точной форме $g_{1} g_{2} \phi_{g_{3}}$ вне $U$; следовательно, форма (6) точна. 
Итак, отображение $g_{1}, g_{2} \mapsto R\left(g_{1}, g_{2}\right)$ есть локальный 2 -коцикл со значениями в $H^{1}(X, \mathbb{R})$. Он продолжается на всю группу $D_{*}\left(X, \omega^{2}\right)$, ибо последняя стягиваема (приложение а)). Получили 2-коцикл $R\left(g_{1}, g_{2}\right), g_{1}, g_{2} \in D_{*}\left(X, \omega^{2}\right)$, со значениями в $H^{1}(X, \mathbb{R})$. Утверждение (а) доказано.

2) Докажем утверждение (b). Пусть для определенности $x_{0} \in Y$. Будем считать, что $U \subset Y$; в противном случае уменьшим области $U_{0}, U$ и окрестность $W$. За счет произвола в выборе формы $\phi_{g}, g \in D^{0}\left(Y, \omega^{2}\right)$, можно считать, что она сосредоточена в $Y$. Заменим в форме (2) отображения $J^{1}, J^{2}$ на $J^{1}-J_{Y}^{1}, J^{2}-J_{Y}^{2}$. Тогда каждое из трех слагаемых в (2) превратится в замкнутую форму; форма $(2)$ изменится только в области $Y$ и, таким образом, определит в $H^{1}(X, \mathbb{R})$ тот же элемент $R\left(g_{1}, g_{2}\right)$. В возникающем слагаемом $g_{1}\left(J^{2}-J_{Y}^{2}\right)\left(\omega^{1} \wedge \phi_{g_{2}}\right)$ (это третье слагаемое из (2)) можно отбросить $g_{1}$ (ибо $g_{1}$ тривиально действует на когомологиях). Далее, рассуждая аналогично предыдущему (т.е. отбрасывая формы, не меняющие класс когомологий), получаем, что элемент $R\left(g_{1}, g_{2}\right), g_{1}, g_{2} \in W_{0}$, представится замкнутой формой $\left(J^{2}-J_{Y}^{2}\right)\left(\omega^{1} \wedge\left(\phi_{g_{1} g_{2}}-\phi_{g_{1}}-\phi_{g_{2}}\right)\right)$. Отсюда $R\left(g_{1}, g_{2}\right)=C_{Y}\left(g_{1} g_{2}\right)-C_{Y}\left(g_{1}\right)-C_{Y}\left(g_{2}\right)$. Теорема 1 доказана.

Теоремы 2 и 3 доказываются в $\$ \$ 4-7$.

\section{§4. Клеточное разбиение поверхности $X$ и разложение диффеоморфизмов в произведение}

Через $X$ обозначается компактная поверхность (без края) рода $>2$ с формой площади $\omega^{2}$; предполагается, что площадь поверхности равна 1 . Группа $D_{*}\left(X, \omega^{2}\right)$ порождается (по ее определению) диффеоморфизмами, сосредоточенными в координатных областях $\sim \mathbb{R}^{2}$. В этом параграфе мы получим достаточно явное разложение элементов, близких к единице, в произведение таких диффеоморфизмов.

4.1. Клеточное разбиение поверхности и соответствующие подгруппы. Зафиксируем стандартное клеточное разбиение $X=\{v\} \cup l_{1} \cup \cdots \cup l_{2 m} \cup U$. Здесь $v$ - точка, $l_{i}, i=1, \ldots, 2 m,-$ интервалы (см. разд. 1.1$), m-$ род поверхности $X, U \sim \mathbb{R}^{2}$. Пусть множества $\bar{l}_{i}=l_{i} \cup\{v\}$ - окружности, трансверсально пересекающиеся в точке $v$.

Введем круг $V \ni v$ и такие области $L_{i} \sim \mathbb{R}^{2}, 1 \leqslant i \leqslant 2 m$, что $l_{i} \subset L_{i}$, $L_{i} \cap L_{j}=\varnothing$ при $i \neq j$. Пусть $\partial L_{i}$ состоит из точки $v$ и двух интервалов с общим концом $v$, причем эти интервалы трансверсально пересекают $\partial V$. Положим $L=$ $\bigcup_{i} L_{i}, \dot{X}=X \backslash\{v\}, \dot{V}=V \backslash\{v\}$.

Метризуем $X$, введя функцию расстояний $\rho$. Взяв число $\varepsilon>0$, положим $V_{\varepsilon}=\{x: x \in V, \rho(x, \partial V)>\varepsilon\} ;$ аналогично определим множества $L_{\varepsilon}, U_{\varepsilon}$. Пусть $\varepsilon$ столь мало, что множества $V_{\varepsilon}, L_{\varepsilon}, U_{\varepsilon}$ покрывают $X$.

Нам понадобятся группы $D^{0}\left(V, \omega^{2}\right), D^{0}\left(L, \omega^{2}\right), D^{0}\left(U, \omega^{2}\right)$; о них сказано в п. с) разд. 1.2. Распространим на группу $D^{0}\left(L, \omega^{2}\right)=\prod_{i} D^{0}\left(L_{i}, \omega^{2}\right)$ отображения $f_{Y}, s_{Y}, C_{Y}, J_{Y}^{2}$. С этой целью для элемента $g=\prod_{i} g_{i}, g_{i} \in D^{0}\left(L_{i}, \omega^{2}\right)$, положим $f_{L}(g)=\prod_{i} f_{L_{i}}\left(g_{i}\right)$ (сомножители перестановочны, а потому порядок их следования безразличен), $s_{L}(g)=\prod_{i} s_{L_{i}}\left(g_{i}\right), C_{L}(g)=\sum_{i} C_{L_{i}}\left(g_{i}\right), J^{2}(g)=\sum_{i} J_{L_{i}}^{2}\left(g_{i}\right)$.

\section{2. Разложение диффеоморфизмов в произведение.}

ПрЕДЛОЖЕние 1 . B группе $D_{*}\left(X, \omega^{2}\right)$ существует окрестность единицьь $W$, обладающая следующим свойством: любой элемент $g \in W$ можно предста- 
вить в виде $g=g_{V} g_{L} g_{U}, g_{V} \in D^{0}\left(V, \omega^{2}\right), g_{L} \in D^{0}\left(L, \omega^{2}\right), g_{U} \in D^{0}\left(U, \omega^{2}\right)$, причем так, что $g_{V}, g_{L}, g_{U}$ непрерывно зависят от $g$ и совпадают с е при $g=e$. Это разложение можно выбрать так, ито $g_{V}, g_{L}, g_{U}$ сосредоточены в областях $V_{\varepsilon}, L_{\varepsilon}, U_{\varepsilon}$ соответственно.

Доказательство будет дано в разд. 4.4.

4.3. Приготовления. а) Рассмотрим группу $D\left(\dot{V}, \omega^{2}\right)$. Возьмем ориентированный интервал $l$, идущий из точки $v$ в некоторую точку из $\partial V$. Величина $\theta(l, g)$, $g \in D\left(\dot{V}, \omega^{2}\right)$ (см. п. а) разд. 1.2$)$ не зависит от выбора интервала $l$; обозначим ее просто через $\theta_{v}(g)$. Легко видеть, что

$$
\beta(\dot{X}, g)-\beta(V, g)=-2 \theta_{v}(g), \quad g \in D\left(\dot{V}, \omega^{2}\right) .
$$

b) Отображения $g \mapsto \beta(V, g), g \mapsto \beta(\dot{X}, g), g \in D\left(\dot{V}, \omega^{2}\right)$, независимы, т. е. для любых двух чисел $a, b \in \mathbb{R}$ найдется такой элемент $g \in D\left(\dot{V}, \omega^{2}\right)$, что $\beta(V, g)=a, \beta(\dot{X}, g)=b$. Действительно, достаточно проверить независимость функций $\beta(V, \cdot), \theta_{v}(\cdot)$. Введем в $V$ координаты $\left(x_{1}, x_{2}\right)$ так, что $V=\left\{\left(x_{1}, x_{2}\right)\right.$ : $\left.x_{1}^{2}+x_{2}^{2}<1\right\}, \omega^{2}=d x_{1} \wedge d x_{2}, v=(0,0)$. Введем полярные координаты $(r, \phi)$. Диффеоморфизм $(r, \phi) \mapsto(r, \phi+f(r)), f \in C_{0}^{\infty}(0,1)$, дает то, что нужно, при соответствующем выборе функции $f \in C_{0}^{\infty}(0,1)$.

c) Справедливы равенства $D_{*}\left(X, \omega^{2}\right) \cap D\left(\dot{X}, \omega^{2}\right)=D_{*}\left(\dot{X}, \omega^{2}\right), D^{0}\left(\dot{X}, \omega^{2}\right) \cap$ $D^{0}\left(V, \omega^{2}\right)=D^{0}\left(\dot{V}, \omega^{2}\right)$.

Первое равенство следует из разд. 1.2. Докажем второе. Пусть $g \in D^{0}\left(\dot{X}, \omega^{2}\right) \cap$ $D^{0}\left(V, \omega^{2}\right)$. Тогда $\beta(\dot{X}, g)=\beta(V, g)=0$ и, согласно $(8), \theta_{v}(g)=0$. Следовательно, $g \in D_{*}\left(\dot{V}, \omega^{2}\right)$. Итак, $g \in D^{0}\left(V, \omega^{2}\right) \cap D_{*}\left(\dot{V}, \omega^{2}\right)=D^{0}\left(\dot{V}, \omega^{2}\right)$. Отсюда следует требуемое.

Следующие утверждения выводятся из работ $[9,10]$.

d) Возьмем круги $U_{i}, i=1,2, \bar{U}_{1} \subset U_{2}$. Пусть диффеоморфизм $g \in D\left(X, \omega^{2}\right)$ отображает замкнутый круг $\bar{U}_{1}$ в $U_{2}$. Тогда существует диффеоморфизм $g^{\prime} \in$ $D\left(U_{2}, \omega^{2}\right)$, совпадающий с $g$ в окрестности круга $\bar{U}_{1}$.

е) Пусть дана область $Y \sim \mathbb{R}^{2}$ и интервал $l$, причем его концы $u, v$ лежат на $\partial Y$ (возможно, $u=v$ ). Пусть дан диффеоморфизм $g \in D\left(X \backslash\{u, v\}, \omega^{2}\right)$, достаточно близкий к единице, и $\theta(l, g)=0$. Тогда существует диффеоморфизм $g^{\prime} \in D\left(Y, \omega^{2}\right)$, совпадающий с $g$ в некоторой окрестности интервала $l$.

\section{4. Доказательство предложения 1.}

Шаг 1. Применяя утверждение d) разд. 4.3 и первое из равенств из п. с) этого раздела, мы можем представить элементы $g \in D_{*}\left(X, \omega^{2}\right)$ из достаточно малой окрестности $W \ni e$ в виде $g=g_{0} h$, где $g_{0} \in D\left(V, \omega^{2}\right), h \in D_{*}\left(\dot{X}, \omega^{2}\right)$.

Шаг 2. Подчиним полученное разложение ограничению $g_{0} \in D^{0}\left(V, \omega^{2}\right), h \in$ $D^{0}\left(\dot{X}, \omega^{2}\right)$. Для этого возьмем элемент $d \in D\left(\dot{V}, \omega^{2}\right)$, для которого $\beta(V, d)=$ $\beta\left(V, g_{0}\right), \beta(\dot{X}, d)=-\beta(\dot{X}, h)$; такой элемент существует согласно утверждению п. b) разд. 4.3. Тогда $\beta\left(V, g_{0} d^{-1}\right)=\beta(\dot{X}, d h)=0$ и, переписав разложение $g=g_{0} h$ в виде $g=g_{0} d^{-1} \cdot d h$, получаем то, что нужно.

Шаг 3. Возьмем $h$ из разложения, указанного в шаге 2 . Так как $h \in D_{*}\left(\dot{X}, \omega^{2}\right)$, то $\theta\left(l_{i}, h\right)=0,1 \leqslant i \leqslant 2 m$. Согласно п. е) разд. 4.3 , существуют диффеоморфизмы $h_{i} \in D\left(L_{i}, \omega^{2}\right)$, совпадающие с $h^{-1}$ в окрестности ребра $l_{i}$. Это приводит к 
разложению

$$
h=\prod_{i=1}^{2 m} h_{i} \cdot g_{2}, \quad g_{2} \in D\left(U, \omega^{2}\right) .
$$

Заметим, что

$$
\sum_{i} \beta\left(L_{i}, h_{i}\right)+\beta\left(U, g_{2}\right)=\beta(\dot{X}, h)=0 .
$$

Шаг 4. Подчиним разложение (9) ограничению $h_{i} \in D^{0}\left(L_{i}, \omega^{2}\right), g_{2} \in D^{0}\left(U, \omega^{2}\right)$ для всех $i=1, \ldots, 2 m$. Для этого возьмем такие элементы $s_{i} \in D\left(X, \omega^{2}\right)$, что $s_{i}$ сосредоточен в области $L_{i} \backslash l_{i}$ и $\beta\left(L_{i}, s_{i}\right)=-\beta\left(L_{i}, h_{i}\right), 1 \leqslant i \leqslant 2 m$. Положим $s=s_{1} \cdots s_{2 m}$. Тогда $\beta\left(L_{i}, h_{i} s_{i}\right)=0, \beta\left(U, s^{-1} g_{2}\right)=0$ (последнее равенство вытекает из (10)). Заменив $h_{i}, g_{2}$ на $h_{i} s_{i}$ и $s^{-1} g_{2}$ соответственно, получаем нужное разложение. Предложение 1 доказано.

\section{§5. Начало доказательства теоремы 2; построение гомоморфизма $F^{\circ}: H^{1}(X, \mathbb{R}) \rightarrow G$}

Разъясним подробнее содержание теоремы 2. Пусть дана произвольная группа $G$ и гомоморфизмы $f_{Y}: D^{0}\left(Y, \omega^{2}\right) \rightarrow G, Y \subset X, Y \sim \mathbb{R}^{2}$, такие, что

$$
f_{Y_{1}}(g)=f_{Y_{2}}(g) \quad \text { при } \bar{Y}_{1} \subset Y_{2}, g \in D^{0}\left(Y_{1}, \omega^{2}\right) \text {. }
$$

Теорема 2 из разд. 2.2 дает основной пример такой ситуации: в качестве $G$ выступает группа $E\left(X, \omega^{2}\right)$, а в качестве $f_{Y}$ выступают отображения $s_{Y}$, заданные формулой (5). Теорема 2 утверждает, что этот пример «универсален»; более точно, существует такой гомоморфизм $F: E\left(X, \omega^{2}\right) \rightarrow G$, что $f_{Y}=F \circ s_{Y}, Y \sim \mathbb{R}^{2}$. Наша цель - построить $F$.

План построения таков. Сначала (в этом параграфе) построим гомоморфизм $F^{\circ}: H^{1}(X, \mathbb{R}) \rightarrow G$, служащий препятствием к продолжению семейства гомоморфизмов $\left\{f_{Y}\right\}$ на всю группу $D_{*}\left(X, \omega^{2}\right)$. Затем (в $\left.\S 6\right)$ продолжим $F^{\circ}$ на всю группу $D_{*}\left(X, \omega^{2}\right)$ (напомним, что группа $H^{1}(X, \mathbb{R})$ вложена в $E\left(X, \omega^{2}\right)$ по правилу $a \mapsto(a, e))$. В результате получится искомый гомоморфизм $F$. Следующий раздел (подготовка к построению отображения $F^{\circ}$ ) - это ядро доказательства теоремы 2 .

5.1. Основная лемма. Для краткости будем называть кольц̧ом любую область в $X$, диффеоморфную кольцу $\left\{(x, y): 1<x^{2}+y^{2}<2\right\}$ на плоскости $\mathbb{R}^{2}$. Образ окружности $x^{2}+y^{2}=3 / 2$ при этом диффеоморфизме назовем направляющей окружностью кольца. Ориентировать кольцо - это выбрать в нем направление обхода, или, что равносильно, ориентировать направляющую окружность.

Возьмем в $X$ две области $A \sim \mathbb{R}^{2}, B \sim \mathbb{R}^{2}$, такие, что множество $A \cap B$ состоит ровно из двух связных компонент $C^{+} \sim \mathbb{R}^{2}, C^{-} \sim \mathbb{R}^{2}$, а множество $A \cup B$ есть кольцо. Ориентируем $A \cup B$ так, что движение внутри области $A$ в указанном направлении ведет от $C^{-}$к $C^{+}$. Рассмотрим группу $D^{0}\left(A, \omega^{2}\right) \cap D^{0}\left(B, \omega^{2}\right)$. Она состоит из диффеоморфизмов вида $g=g^{-} g^{+}$, где $g^{-} \in D\left(C^{-}, \omega^{2}\right), g^{+} \in D\left(C^{+}, \omega^{2}\right)$, $\beta\left(C^{-}, g^{-}\right)+\beta\left(C^{+}, g^{+}\right)=0$. Всюду в дальнейшем запись $g=g^{-} g^{+}$имеет именно такой смысл, и мы не будем более пояснять это. Отображения $f_{A}, f_{B}$, рассматриваемые на $D^{0}\left(A, \omega^{2}\right) \cap D^{0}\left(B, \omega^{2}\right)$, не обязаны совпадать, и мы изучим сейчас «различающее» их отображение $g \mapsto f_{A}(g) f_{B}\left(g^{-1}\right), g \in D^{0}\left(A, \omega^{2}\right) \cap D^{0}\left(B, \omega^{2}\right)$. 
ЛЕмма 1 (основная). (а) Существует такой гомоморфизм $\tau: \mathbb{R} \rightarrow G$, ито $f_{A}(g) f_{B}\left(g^{-1}\right)=\tau\left(\beta\left(C^{-}, g^{-}\right)\right), g=g^{-} g^{+} \in D^{0}\left(A, \omega^{2}\right) \cap D^{0}\left(B, \omega^{2}\right)$. (Таким образом, указанное "различающее» отображение есть гомоморфизм групп.)

(b) Элемент $\tau(x), x \in \mathbb{R}$, перестановочен с любым элементом $f_{Y}(h)$, где $h \in D^{0}\left(Y, \omega^{2}\right), Y \sim \mathbb{R}^{2}$.

(с) Отображение $\tau$ зависит только от класса гомотопий ориентированного кольцза $A \cup B$.

ДОКАЗАТЕЛЬСТВО.

Шаг 1. Пусть $g=g^{-} g^{+} \in D^{0}\left(A, \omega^{2}\right) \cap D^{0}\left(B, \omega^{2}\right), \beta\left(C, g^{-}\right)=0$. (Таким образом, $g^{-}, g^{+} \in D^{0}\left(A, \omega^{2}\right) \cap D^{0}\left(B, \omega^{2}\right)$.) Докажем, что $f_{A}(g)=f_{B}(g)$. Действительно, $f_{A}(g)=f_{A}\left(g^{-}\right) f_{A}\left(g^{+}\right)=f_{C^{-}}\left(g^{-}\right) f_{C^{+}}\left(g^{+}\right)$. Аналогично получаем равенство $f_{B}(g)=f_{C^{-}}\left(g^{-}\right) f_{C^{+}}\left(g^{+}\right)$. Итак, $f_{A}(g)=f_{B}(g)$, что и требовалось.

Шаг 2. Пусть $g, h \in D^{0}\left(A, \omega^{2}\right) \cap D^{0}\left(B, \omega^{2}\right), \beta\left(C^{-}, g^{-}\right)=\beta\left(C^{-}, h^{-}\right)$. Тогда $f_{A}(g) f_{B}\left(g^{-1}\right)=f_{A}(h) f_{B}\left(h^{-1}\right)$. Для доказательства достаточно применить шаг 1 к элементу $h^{-1} g$.

Из доказанного сейчас вытекает существование такого отображения $\tau: \mathbb{R} \rightarrow$ $G$, что $f_{A}(g) f_{B}\left(g^{-1}\right)=\tau\left(\beta\left(C^{-}, g^{-}\right)\right)$.

Шаг 3. Докажем, что отображение $\tau$ - гомоморфизм. Возьмем числа $x, y$ и такие элементы $g, h \in D^{0}\left(A, \omega^{2}\right) \cap D^{0}\left(B, \omega^{2}\right)$, что $\beta\left(C^{-}, g^{-}\right)=x, \beta\left(C^{-}, h^{-}\right)=y$. Пусть, кроме того, элементы $h, g$ имеют непересекающиеся носители, заключенные в областях $A_{1} \subset A, B_{1} \subset B$, причем области $A_{1}, B_{1}, D=A_{1} \cup B_{1}$ диффеоморфны плоскости $\mathbb{R}^{2}$. Покажем, что элементы $f_{A}(h), f_{B}(g)$ перестановочны. Действительно, из свойства (11) следует, что $f_{A}(h)=f_{A_{1}}(h)=f_{D}(h)$ и, аналогично, $f_{B}(g)=f_{D}(g)$; отсюда вытекает упомянутая перестановочность. Теперь получаем

$$
\begin{aligned}
\tau(x) \tau(y) & =f_{A}(g) f_{B}\left(g^{-1}\right) f_{A}(h) f_{B}\left(h^{-1}\right)=f_{A}(g) f_{A}(h) f_{B}\left(g^{-1}\right) f_{B}\left(h^{-1}\right) \\
& =f_{A}(h g) f_{B}\left((h g)^{-1}\right)=\tau\left(\beta\left(C^{-}, h^{-} g^{-}\right)\right)=\tau\left(\beta\left(C^{-}, g^{-}\right)+\beta\left(C^{-}, h^{-}\right)\right) \\
& =\tau(x+y),
\end{aligned}
$$

что и требовалось.

Мы доказали утверждение (а) леммы 1. Из него следует утверждение (c).

Шаг 4. Докажем утверждение (b). Достаточно сделать это для диффеоморфизма $h$ со сколь угодно малым носителем. Пользуясь утверждениями (а), (c) (меняя области $A, B)$, можно считать, что $Y \cap(A \cup B)=\varnothing$. Тогда утверждение (b) становится очевидным. Лемма доказана.

Эта лемма мотивирует следующее определение. Возьмем в $X$ ориентированную окружность $r$ (см. разд. 1.1), окружим ее кольцом с соответствующей ориентацией и возьмем гомоморфизм $\tau$, как в лемме 1 . Он не зависит от выбора кольца и не меняется при деформации окружности $r$. Обозначим его через $\tau_{r}$. Теперь утверждение леммы 1 запишется в виде

$$
f_{A}(g) f_{B}\left(g^{-1}\right)=\tau_{r}\left(\beta\left(C^{-}, g^{-}\right)\right), \quad g \in D^{0}\left(A, \omega^{2}\right) \cap D^{0}\left(B, \omega^{2}\right),
$$

где $r$ - направляющая окружность в кольце $A \cup B$.

Применим построения леммы 1 к нашему основному примеру $-\mathrm{k}$ группе $E\left(X, \omega^{2}\right)$ и семейству отображений $\left\{s_{Y}, Y \sim \mathbb{R}^{2}\right\}$. Положим $\Gamma_{A B}(g)=C_{A}(g)-$ $C_{B}(g), g \in D^{0}\left(A, \omega^{2}\right) \cap D^{0}\left(B, \omega^{2}\right)$. (Отображения $C_{Y}$ были введены в разд. 2.2.) 
Согласно разд. 2.2,

$$
s_{A}(g) s_{B}\left(g^{-1}\right)=\Gamma_{A B}(g), \quad g \in D^{0}\left(A, \omega^{2}\right) \cap D^{0}\left(B, \omega^{2}\right) .
$$

Чтобы найти для этого случая отображение $\tau_{r}$, возьмем в кольце $A \cup B$ ориентированный интервал $l$, такой, что его концы лежат на границе кольца и индекс пересечения $[l, r]$ равен 1. Согласно разд. 2.2, элемент $\Gamma_{A B}(g)$, $g \in D^{0}\left(A, \omega^{2}\right) \cap D^{0}\left(B, \omega^{2}\right)$, представляется 1-формой $\left(J_{B}-J_{A}\right)\left(\omega^{1} \wedge \phi_{g}\right)$. Имеем равенство

$$
\left\langle\Gamma_{A B}(g), l\right\rangle=\beta\left(C^{-}, g^{-}\right),
$$

где левая часть (спаривание) определяется как интеграл упомянутой формы по $l$. (Действительно, считая для упрощения, что $l \subset B \backslash A$, преобразуем левую часть к виду $\int_{l}\left(J_{B}-J_{A}\right)\left(\omega^{1} \wedge \phi_{g}\right)=\int_{C^{-}} \omega^{1} \wedge g \omega^{1}=\beta\left(C^{-}, g^{-}\right)$.) Таким образом, элемент $\tau_{r}(x) \in H^{1}(X, \mathbb{R}), x \in \mathbb{R}$, представляется такой 1 -формой $\xi^{1} \in Z_{1}(A \cup B)$, что $\int_{l} \xi^{1}=x$.

5.2. Построение гомоморфизма $\boldsymbol{F}^{\circ}: \boldsymbol{H}^{\mathbf{1}}(\boldsymbol{X}, \mathbb{R}) \rightarrow G$. При построении клеточного разбиения (разд. 4.1) возникли интервалы $l_{i}$ и окружности $\bar{l}_{i}, 1 \leqslant i \leqslant 2 m$. Ориентировав $\bar{l}_{i}$, получаем базис в линейном пространстве $H_{1}(X, \mathbb{R})$.

Для любого $i=1, \ldots, 2 m$ множество $U \cup l_{i}$ диффеоморфно кольцу; следуя соглашению из разд. 5.1, назовем его кольцом. Возьмем в нем ориентированную окружность $r_{i}$, такую, что индекс пересечения $\left[l_{i}, r_{i}\right]$ равен +1 .

Определим отображение

$$
F^{\circ}: H^{1}(X, \mathbb{R}) \rightarrow G, \quad F^{\circ}(a)=\prod_{i=1}^{2 m} \tau_{r_{i}}\left(\left\langle a, \bar{l}_{i}\right\rangle\right) .
$$

(Оно мотивировано построениями из разд. 5.1.) Здесь $\left\langle a, \bar{l}_{i}\right\rangle$ означает естественное спаривание между элементом $a \in H^{1}(X, \mathbb{R})$ и 1 -циклом $\bar{l}_{i}$.

Это и есть обещанное в начале этого параграфа препятствие к продолжению гомоморфизмов $\left\{f_{Y}\right\}$ на всю группу $D_{*}\left(X, \omega^{2}\right)$. По лемме 1 элемент $F^{\circ}(a), a \in$ $H^{1}(X, \mathbb{R})$, перестановочен с $f_{Y}(g)$ для любого $g \in D^{0}\left(Y, \omega^{2}\right), Y \sim \mathbb{R}^{2}$.

В следующем параграфе мы покажем, что $F^{\circ}$ можно продолжить до гомоморфизма $F: E\left(X, \omega^{2}\right) \rightarrow G$, о котором говорилось в начале этого параграфа.

\section{§6. Окончание доказательства теоремы 2; построение гомоморфизма $F: E\left(X, \omega^{2}\right) \rightarrow G$}

Для любой области $Y \subset X$ с конечным числом связных компонент имеем группу $D^{0}\left(Y, \omega^{2}\right) \subset D_{*}\left(X, \omega^{2}\right)$ (см. п. с) разд. 1.2$)$ и ее прообраз $\widetilde{D}^{0}\left(Y, \omega^{2}\right)=$ $\pi^{-1}\left(D^{0}\left(Y, \omega^{2}\right)\right) \subset E\left(X, \omega^{2}\right)$. Группа $E\left(X, \omega^{2}\right)$ порождена подгруппами $\widetilde{D}^{0}\left(V, \omega^{2}\right)$, $\widetilde{D}^{0}\left(L, \omega^{2}\right), \widetilde{D}^{0}\left(U, \omega^{2}\right)$. Будем последовательно строить искомый гомоморфизм $F$ - сначала на этих подгруппах и, наконец, на всей группе $E\left(X, \omega^{2}\right)$. (Пока мы определили его лишь на центральной подгруппе $H^{1}(X, \mathbb{R})$.)

Мы отождествляем элементы $(e, a) \in E\left(X, \omega^{2}\right)$ и $a \in H^{1}\left(X, \omega^{2}\right)$.

6.1. Некоторые соотношения. Каждая область $L_{i} \backslash l_{i}$ состоит из двух связных частей $L_{i}^{-}, L_{i}^{+}$; пусть $L_{i}^{-}$лежит слева при движении по $l_{i}$ согласно ориентации. 
Рассмотрим группу $D^{0}\left(L, \omega^{2}\right) \cap D^{0}\left(U, \omega^{2}\right)$. Она состоит из диффеоморфизмов вида

где

$$
g=\prod_{i} g_{i}, \quad g_{i}=g_{i}^{-} g_{i}^{+},
$$

$$
g_{i}^{-} \in D\left(L_{i}^{-}, \omega^{2}\right), \quad g_{i}^{+} \in D\left(L_{i}^{+}, \omega^{2}\right), \quad \beta\left(L_{i}^{-}, g_{i}^{-}\right)+\beta\left(L_{i}^{+}, g_{i}^{+}\right)=0 .
$$

Имеем гомоморфизмы $f_{L}, f_{U}$ из нее в $G$, а также гомоморфизмы $s_{L}, s_{U}$ из нее в $E\left(X, \omega^{2}\right)$. Изучим «различающие» отображения $g \mapsto f_{U}(g) f_{L}\left(g^{-1}\right), g \mapsto$ $s_{U}(g) s_{L}\left(g^{-1}\right), g \in D^{0}\left(L, \omega^{2}\right) \cap D^{0}\left(U, \omega^{2}\right)$. Для этого введем отображение

$$
\Gamma: D^{0}\left(L, \omega^{2}\right) \cap D^{0}\left(U, \omega^{2}\right) \rightarrow H^{1}(X, \mathbb{R}), \quad \Gamma(g)=C_{U}(g)-C_{L}(g) .
$$

Следующая лемма описывает упомянутые «различающие» отображения.

ЛЕмма 2. Пусть $g \in D^{0}\left(L, \omega^{2}\right) \cap D^{0}\left(U, \omega^{2}\right)$. Тогда

$$
\begin{aligned}
& f_{U}(g) f_{L}\left(g^{-1}\right)=F^{\circ}(\Gamma(g)), \\
& s_{U}(g) s_{L}\left(g^{-1}\right)=\Gamma(g) .
\end{aligned}
$$

(Напомним, что в последнем равенстве элемент $\Gamma(g) \in H^{1}(X, \mathbb{R})$ отождествляется с $(e, \Gamma(g)) \in E\left(X, \omega^{2}\right)$.)

Доказательство. Равенство (20) немедленно следует из того, что $s_{U}(g)=$ $\left(g, C_{U}(g)\right), s_{L}(g)=\left(g, C_{L}(g)\right), g \in D^{0}\left(L, \omega^{2}\right) \cap D^{0}\left(U, \omega^{2}\right)$.

Чтобы доказать равенство (19), зафиксируем один сомножитель $g_{i}$ из произведения (16). Применим построения из разд. 5.1, полагая $A=U, B=L_{i}$. Как уже отмечалось, область $U \cup L_{i}=U \cup l_{i}$ есть кольцо с направляющей окружностью $r_{i}$.

Равенства (12), (14) перепишутся в виде

$$
\begin{aligned}
f_{U}\left(g_{i}\right) f_{L_{i}}\left(g_{i}^{-1}\right) & =\tau_{r_{i}}\left(\beta\left(L_{i}^{-}, g_{i}^{-}\right)\right), \\
\left\langle\Gamma\left(g_{i}\right), \bar{l}_{i}\right\rangle & =\beta\left(L_{i}^{-}, g_{i}^{-}\right),
\end{aligned}
$$

$g_{i} \in D^{0}\left(L_{i}, \omega^{2}\right) \cap D^{0}\left(U, \omega^{2}\right)$.

Теперь для доказательства равенства (19) возьмем элемент $g \in D^{0}\left(L, \omega^{2}\right) \cap$ $D^{0}\left(U, \omega^{2}\right)$ и представим его в виде $(16),(17)$. Из того, что элемент $f_{U}\left(g_{i}\right) f_{L_{i}}\left(g_{i}^{-1}\right)$ перестановочен с любым $f_{Y}(g), g \in D^{0}\left(Y, \omega^{2}\right), Y \sim \mathbb{R}^{2}$, легко следует, что

$$
f_{U}(g) f_{L}\left(g^{-1}\right)=\prod_{i=1}^{2 m}\left(f_{U}\left(g_{i}\right) f_{L_{i}}\left(g_{i}^{-1}\right)\right) .
$$

Используя равенства (21), (22), а затем равенство (15), приводим последнее выражение к виду

$$
\prod_{i=1}^{2 m} \tau_{r_{i}}\left(\beta\left(L_{i}^{-}, g_{i}^{-}\right)\right)=\prod_{i=1}^{2 m} \tau_{r_{i}}\left(\left\langle\Gamma\left(g_{i}\right), \bar{l}_{i}\right\rangle\right)=F^{\circ}(\Gamma(g)) .
$$

Этим доказано (19). Лемма доказана.

6.2. Построение гомоморфизмов $\boldsymbol{F}_{\boldsymbol{V}}, \boldsymbol{F}_{\boldsymbol{L}}, \boldsymbol{F}_{\boldsymbol{U}}$. Введем отображения

$$
\begin{aligned}
F_{V}: \widetilde{D}^{0}\left(V, \omega^{2}\right) \rightarrow G, & F_{V}(\gamma)=f_{V}(g) F^{\circ}\left(a-C_{V}(g)\right), \\
F_{L}: \widetilde{D}^{0}\left(L, \omega^{2}\right) \rightarrow G, & F_{L}(\gamma)=f_{L}(g) F^{\circ}\left(a-C_{L}(g)\right), \\
F_{U}: \widetilde{D}^{0}\left(U, \omega^{2}\right) \rightarrow G, & F_{U}(\gamma)=f_{U}(g) F^{\circ}\left(a-C_{U}(g)\right) .
\end{aligned}
$$


(Здесь $\gamma=(g, a)$.

Легко видеть, что это гомоморфизмы, и если $Y \subset V, Y \sim \mathbb{R}^{2}$, то $f_{Y}=$ $F_{V} \circ s_{Y}$. Аналогичное утверждение справедливо и для отображений $F_{L}, F_{U}$.

ЛЕмма 3. $F_{L}(\gamma)=F_{U}(\gamma) n p u \gamma \in \widetilde{D}^{0}\left(L, \omega^{2}\right) \cap \widetilde{D}^{0}\left(U, \omega^{2}\right)$.

ДоказАТЕЛьство. Пусть $\gamma \in \widetilde{D}^{0}\left(L, \omega^{2}\right) \cap \widetilde{D}^{0}\left(U, \omega^{2}\right)$. Тогда элементы $F_{L}(\gamma)$, $F_{U}(\gamma)$, заданные формулами $(24),(25)$, равны в силу равенств (18), (19). Лемма доказана.

6.3. Построение гомоморфизма $\boldsymbol{F}$. Построим теперь локальный гомоморфизм $F_{\dot{X}}: \widetilde{D}^{0}\left(\dot{X}, \omega^{2}\right) \rightarrow G$ следующим образом. По предложению 1 любой элемент $\gamma=(g, a) \in \widetilde{D}^{0}\left(\dot{X}, \omega^{2}\right)$, где $g$ близок к единице, представляется в виде произведения $\gamma=\gamma_{L} \gamma_{U}, \gamma_{L} \in \widetilde{D}^{0}\left(L, \omega^{2}\right), \gamma_{U} \in \widetilde{D}^{0}\left(U, \omega^{2}\right)$. Положим $F_{\dot{X}}(\gamma)=$ $F_{L}\left(\gamma_{L}\right) F_{U}\left(\gamma_{U}\right)$. Из леммы 3 следует, что $F_{\dot{X}}(\gamma)$ не зависит от выбора разложения в произведение элемента $\gamma$. Докажем, что $F_{\dot{X}}(\gamma) F_{\dot{X}}(\delta)=F_{\dot{X}}(\gamma \delta), \gamma=(g, a)$, $\delta=(d, b)$, где $g=\pi(\gamma), d=\pi(\delta)$ близки к единице. Для этого выберем разложения $\gamma=\gamma_{L} \gamma_{U}, \delta=\delta_{L} \delta_{U}$ так, что носители элементов $\pi\left(\gamma_{U}\right), \pi\left(\delta_{L}\right)$ не пересекаются; это можно сделать в силу второго утверждения предложения 1. Отсюда легко выводится, что элементы $\gamma_{U}, \delta_{L}$ перестановочны; перестановочны также $F_{U}\left(\gamma_{U}\right), F_{L}\left(\delta_{L}\right)$. Но тогда $F_{\dot{X}}(\gamma) F_{\dot{X}}(\delta)=F_{L}\left(\gamma_{L}\right) F_{U}\left(\gamma_{U}\right) F_{L}\left(\delta_{L}\right) F_{U}\left(\delta_{U}\right)=$ $F_{L}\left(\gamma_{L} \delta_{L}\right) F_{U}\left(\gamma_{U} \delta_{U}\right)=F_{\dot{X}}(\gamma \delta)$. Итак, $F_{\dot{X}}-$ локальный гомоморфизм.

ЛЕМма 4. $F_{V}(\gamma)=F_{\dot{X}}(\gamma) n p u \gamma \in \widetilde{D}^{0}\left(V, \omega^{2}\right) \cap \widetilde{D}^{0}\left(\dot{X}, \omega^{2}\right)$.

ДоказАТельство. Пусть $\gamma=(g, a)$. Таким образом, $g \in D^{0}\left(V, \omega^{2}\right) \cap D^{0}\left(\dot{X}, \omega^{2}\right)$. Согласно п. с) разд. $4.3, g \in D^{0}\left(\dot{V}, \omega^{2}\right)$. Поэтому достаточно доказать лемму для $g \in D^{0}\left(Y, \omega^{2}\right)$, где $Y \sim \mathbb{R}^{2}, Y \subset \dot{V}$. Но тогда $F_{V}(\gamma)=F_{Y}(\gamma), F_{\dot{X}}(\gamma)=F_{Y}(\gamma)$, чем доказана лемма.

Построим локальный гомоморфизм $F: E\left(X, \omega^{2}\right) \rightarrow G$. Возьмем элемент $\gamma \in$ $E\left(X, \omega^{2}\right), \gamma=(g, a)$, где $g$ близок к единице. Согласно предложению $1, \gamma=$ $\gamma_{V} \gamma_{\dot{X}}, \gamma_{V} \in \widetilde{D}^{0}\left(V, \omega^{2}\right), \gamma_{\dot{X}} \in \widetilde{D}^{0}\left(\dot{X}, \omega^{2}\right)$. Положим $F(\gamma)=F_{V}\left(\gamma_{V}\right) F_{\dot{X}}\left(\gamma_{\dot{X}}\right)$. Тот факт, что $F(\gamma)$ не зависит от выбора разложения элемента $\gamma$, вытекает из леммы 4. Для доказательства равенства $F(\gamma \delta)=F(\gamma) F(\delta)$ выбираем разложения $\gamma=\gamma_{V} \gamma_{\dot{X}}, \delta=\delta_{V} \delta_{\dot{X}}$ так, что $\pi\left(\gamma_{\dot{X}}\right), \pi\left(\delta_{V}\right)$ имеют непересекающиеся носители.

Итак, построен локальный гомоморфизм $F: E\left(X, \omega^{2}\right) \rightarrow G$. Так как группа $E\left(X, \omega^{2}\right)$ стягиваема, то он продолжается на всю группу. Если $Y \subset X, Y \sim \mathbb{R}^{2}$, то $F \circ s_{Y}=f_{Y}$. (Это очевидно для областей $Y$, целиком лежащих в одной из областей $V, L, U$; но тогда это верно и для любой области $Y$, ибо $D^{0}\left(Y, \omega^{2}\right)$ порождается диффеоморфизмами со сколь угодно малыми носителями.) Теорема 2 доказана.

\section{§7. Доказательство теоремы 3}

Дана группа $G$ и гомоморфизмы $\phi_{Y}: D\left(Y, \omega^{2}\right) \rightarrow G, Y \subset X, Y \sim \mathbb{R}^{2}$, причем $\phi_{Y_{1}}(g)=\phi_{Y_{2}}(g)$ при $g \in D\left(Y_{1}, \omega^{2}\right), \bar{Y}_{1} \subset Y_{2}$. Требуется построить такой гомоморфизм $\Phi: D_{*}\left(X, \omega^{2}\right) \times \mathbb{R} \rightarrow A$, что $\phi_{Y}(g)=\Phi(g, \beta(Y, g))$ при $g \in D\left(Y, \omega^{2}\right)$, $Y \sim \mathbb{R}^{2}$

Рассуждаем, как в разд. 6.3. Но здесь все проще, и удается продолжить гомоморфизмы $\left\{\phi_{Y}, Y \subset \dot{X}, Y \sim \mathbb{R}^{2}\right\}$ до локального гомоморфизма $\phi_{\dot{X}}: D_{*}\left(\dot{X}, \omega^{2}\right) \rightarrow G$. 
Кроме того, имеем гомоморфизм $\phi_{V}: D\left(V, \omega^{2}\right) \rightarrow G$. Однако эти два гомоморфизма не обязаны совпадать на подгруппе $D\left(\dot{V}, \omega^{2}\right)$. Возникает «различающее» отображение $g \mapsto \phi_{\dot{X}}(g) \phi_{V}\left(g^{-1}\right), g \in D\left(\dot{V}, \omega^{2}\right)$. Рассуждая по аналогии с разд. 5.1, получаем следующее:

a) существует такой гомоморфизм $\sigma: \mathbb{R} \rightarrow G$, что $\phi_{\dot{X}}(g) \phi_{V}\left(g^{-1}\right)=\sigma\left(\theta_{v}(g)\right)$, $g \in D\left(\dot{V}, \omega^{2}\right)$,

b) если $Y \subset X, Y \sim \mathbb{R}^{2}, g \in D\left(Y, \omega^{2}\right), t \in \mathbb{R}$, то элементы $\phi_{Y}(g), \sigma(t)$ перестановочны.

Из равенства (8) видно, что «подправленные» гомоморфизмы $g \mapsto \phi_{V}(g) \sigma(\beta(V, g))$, $g \mapsto \phi_{\dot{X}}(g) \sigma(\beta(\dot{X}, g))$ совпадают на $D\left(\dot{V}, \omega^{2}\right)$. Поэтому существует гомоморфизм $S: D_{*}\left(X, \omega^{2}\right) \rightarrow G$, продолжающий эти два гомоморфизма. Гомоморфизм $\Phi: D_{*}\left(X, \omega^{2}\right) \times \mathbb{R} \rightarrow G,(g, a) \mapsto S(g) \sigma(-a)$, завершает доказательство теоремы 3.

\section{§8. Некоторые следствия: образующие и соотношения в группе $D_{*}\left(X, \omega^{2}\right)$, точные последовательности}

8.1. Образующие и соотношения в группе $D_{*}\left(X, \omega^{2}\right)$. Рассмотрим группу $\Sigma$, заданную образующими и соотношениями следующим образом. Образующие - это всевозможные пары $(Y, g)$, где $Y \subset X, Y \sim \mathbb{R}^{2}, g \in D\left(Y, \omega^{2}\right)$. Соотношения таковы: 1) $\left(Y_{1}, g\right)=\left(Y_{2}, g\right)$ при $\left.g \in D\left(Y_{1}, \omega^{2}\right), \bar{Y}_{1} \subset Y_{2} ; 2\right)\left(Y, g_{1}\right)\left(Y, g_{2}\right)^{-1}$ $=\left(Y, g_{1} g_{2}^{-1}\right)$ при $g_{1}, g_{2} \in D\left(Y, \omega^{2}\right)$. (Менее формально: образующие - это диффеоморфизмы, сосредоточенные в областях $\sim \mathbb{R}^{2}$, а соотношения - в точности те соотношения между элементами группы $D\left(X, \omega^{2}\right)$, которые можно записать, оставаясь в областях $\sim \mathbb{R}^{2}$.) Теорема 3 утверждает, что $\Sigma \sim D_{*}\left(X, \omega^{2}\right) \times \mathbb{R}$; при этом образующая $(Y, g)$ вкладывается в $\Sigma$ следующим образом: $(Y, g) \mapsto$ $(g, \beta(Y, g))$. Чтобы уничтожить «лишний» прямой сомножитель $\mathbb{R}$ (и получить группу $\left.D_{*}\left(X, \omega^{2}\right)\right)$, нужны добавочные соотношения; во введении мы назвали их «глобальными» (по контрасту с указанными выше «локальными»). Сейчас мы выпишем их явно. Используем обозначения из разд. 4.1.

Возьмем такие $g(t) \in D\left(\dot{V}, \omega^{2}\right), t \in \mathbb{R}$, что $\theta_{v}(g(t))=t, g_{0}=e$ (см. п. b) разд. 3.1) и соответствие $t \mapsto g(t)$ непрерывно. Пусть $\varepsilon>0$ столь мало, что $l_{i} g(t) \subset L_{i}$ при $|t|<\varepsilon, i=1, \ldots, 2 m$. Ясно, что $\theta\left(l_{i}, g(t)\right)=0$; поэтому, согласно п. е) разд. 4.3 , существует элемент $g_{i}(t) \in D\left(L_{i}, \omega^{2}\right)$, совпадающий с $g(t)$ в окрестности дуги $l_{i}$. Это приводит к разложению (в духе разд. 4.2) вида $g(t)=h(t) \prod_{i=1}^{2 m} g_{i}(t), h(t) \in D\left(U, \omega^{2}\right)$. Теперь искомые добавочные (глобальные) соотношения для образующих можно задать в виде

$$
(U, h(t))\left(L_{1}, g_{1}(t)\right) \cdots\left(L_{2 m}, g_{2 m}(t)\right)\left(V,\left(g(t)^{-1}\right)=e, \quad t \in(-\varepsilon, \varepsilon) .\right.
$$

Действительно, при отображении свободной группы, порожденной образующими $(Y, g)$, на группу $\Sigma \sim D_{*}\left(X, \omega^{2}\right) \times \mathbb{R}$ левая часть равенства $(26)$ переходит в

$$
\begin{gathered}
\left(e, \beta(U, h(t))+\sum_{i} \beta\left(L_{i}, g_{i}(t)\right)-\beta(V, g(t))\right)(e, \beta(\dot{X}, g(t))-\beta(V, g(t))) \\
=\left(e,-2 \theta_{v}(g(t))\right)=(e,-2 t)
\end{gathered}
$$

здесь использовано соотношение (8). Наложение соотношений (26) заменяет $(e,-2 t)$ на $e$. Отсюда следует требуемое.

Аналогичное истолкование допускает и теорема 2. 
8.2. Точные последовательности. Мы рассмотрели группы $D\left(X, \omega^{2}\right) \supset$ $D_{*}\left(X, \omega^{2}\right)$ и центральное расширение последней. Имеем точную последовательность

$$
0 \rightarrow D_{*}\left(X, \omega^{2}\right) \rightarrow D\left(X, \omega^{2}\right) \rightarrow H^{1}(X, \mathbb{R}) \rightarrow 0,
$$

(см. п. d) разд. 1.2). Соединив ее с (4), получаем длинную точную последовательность

$$
0 \rightarrow H^{1}(X, \mathbb{R}) \rightarrow E\left(X, \omega^{2}\right) \rightarrow D\left(X, \omega^{2}\right) \rightarrow H^{1}(X, \mathbb{R}) \rightarrow 0 .
$$

Эти построения (группа, ее фактор по подгруппе, порожденной «малыми» подгруппами, центральное расширение последней, ... ) внешне напоминают известные конструкции алгебраической $K$-теории.

\section{Приложение}

а) Стягиваемость группы $\boldsymbol{D}_{*}\left(\boldsymbol{X}, \boldsymbol{\omega}^{\mathbf{2}}\right)$. Группа $D(X)$ (связная компонента единицы в группе всех диффеоморфизмов) стягиваема при выполнении условия $(*)[11]$. Имеется ретракция $D(X) \rightarrow D\left(X, \omega^{2}\right)$, построенная, по существу, Ю. Мозером [9]; этот круг вопросов изложен и развит также в [10]. Следовательно, $D\left(X, \omega^{2}\right)$ стягиваема. Далее, отображение $\alpha: D\left(X, \omega^{2}\right) \rightarrow H^{1}(X, \mathbb{R})$ из п. d) разд. 1.2 допускает правое обратное. Чтобы построить его, возьмем в $X$ ориентированные окружности $r_{i}, 1 \leqslant i \leqslant 2 m(m-$ род поверхности), задающие базис в $H_{1}(X, \mathbb{R})$. Каждую окружность $r_{i}$ заключим в узкую полоску $R_{i}$ и возьмем в ней ориентированный интервал $w_{i}$, соединяющий граничные окружности. Действуя, как в п. а) разд. 4.3 , нетрудно построить такой гомоморфизм $\Psi_{i}: \mathbb{R} \rightarrow D\left(R_{i}, \omega^{2}\right)$, что $\theta\left(w_{i}, \Psi_{i}(t)\right)=t, t \in \mathbb{R}$. Отображение $\left(t_{1}, \ldots, t_{2 m}\right) \mapsto \Psi_{1}\left(t_{1}\right) \cdots \Psi_{2 m}\left(t_{2 m}\right)$ и есть искомое правое обратное к $\alpha: D\left(X, \omega^{2}\right) \rightarrow H^{1}(X, \mathbb{R})$ (здесь $\left\{t_{i}\right\}$ - координаты в пространстве $H^{1}(X, \mathbb{R})$ по отношению к подходящему базису). Отсюда следует стягиваемость группы $D_{*}\left(X, \omega^{2}\right)=\operatorname{ker} \alpha$.

b) Об отображении $\boldsymbol{J}^{\mathbf{2}}: \boldsymbol{B}^{\mathbf{2}}(\boldsymbol{X}) \rightarrow \boldsymbol{E}^{\mathbf{1}}(\boldsymbol{X})$. Фиксируем круги $U_{i}, i=1,2$, $\bar{U}_{1} \subset U_{2}$. Требуется построить такое отображение $J^{2}$ (линейное непрерывное), что $d \circ J^{2}(\xi)=\xi, \xi \in B^{2}(X), J^{2}\left(B^{2}\left(U_{1}\right)\right) \subset E^{1}\left(U_{2}\right)$. Для этого возьмем какиелибо отображения $M: B^{2}(X) \rightarrow E^{1}(X), N: B^{2}\left(U_{2}\right) \rightarrow E^{1}\left(U_{2}\right)$, обратные справа к $d$, функцию $\phi \in C^{\infty}(X)$, равную 1 на $U_{1}$ и 0 вне $U_{2}$, и 2 -форму $\tau_{0} \in E^{2}\left(U_{1}\right)$, $\int_{X} \tau_{0}=1$. Положим $J^{2}(\xi)=M\left((1-\phi) \xi+\left(\int_{X} \phi \xi\right) \tau_{0}\right)+N\left(\phi \xi-\left(\int_{X} \phi \xi\right) \tau_{0}\right)$. Это и есть искомое отображение.

\section{ЛИТЕРАТУРА}

1. Серр Ж. П. Амальгамы, деревья и $S L_{2}$. Сб. переводов «Математика», 18:1, 3-51 (1974).

2. Calabi E. Problems in Analysis. Symp. in honor of S. Bochner, Princeton University Press, Princeton, NY, 1970, pp. 1-26.

3. Арнольд В. И. Об одномерных когомологиях алгебры Ли бездивергентных векторных полей и о числах вращения динамических систем. Функц. анализ и его прил., $\mathbf{3}$, вып. 4, 77-78 (1969).

4. Banyaga A. Comment. Math. Helv., 53, No. 2, 174-227 (1978).

5. McDuff $D$. The homology of some groups of diffeomorphisms. Comment. Math. Helv., 55, 97-129 (1980). 
6. Kirillov A. A. La géométrie des moments pour les groups de difféomorphismes. Operator algebras, Unitary Representations, Enveloping Algebras, and Invariant Theory, Progress in Mathematics, Vol. 92, 1990.

7. Khesin B., Lyubashenko V., Roger C. Extensions and contractions of the Lie algebra of $q$-pseudodifferential symbols on the circle. J. Funct. Anal., 143, No. 1, 55-97 (1997).

8. Van Est W. T. Local and global groups. Indag. Math., 24, 391-425 (1962).

9. Moser J. On the volume elements on a manifold. Trans. Amer. Math. Soc., 120, 286-294 (1965).

10. Крыгин А. Б. Продолжение диффеоморфизмов, сохраняющих объем. Функц. анализ и его прил., 5, вып. 2, 72-76 (1971).

11. Earle C. J., Eells J. jr. The diffeomorphism group of a compact Riemann surface. Bull. Amer. Math. Soc., 73, 557-559 (1967).

12. Исмагилов P. С. О группе диффеоморфизмов, сохраняющих объем. Изв. АН СССР, 44, № 4, 831-867 (1980).

13. Исмагилов Р. С. Примеры явного вычисления индуктивного предела семейств алгебр Ли. Матем. сб., 191, № 3, 53-64 (2000).

Московский государственный

технический университет им. Н. Э. Баумана

Поступило в редакцию 11 февраля 2002 г. 